\title{
Optimal Approximation of Biquartic Polynomials by Bicubic Splines
}

\author{
Viliam Kačala ${ }^{1, \star}$ and Csaba Török ${ }^{1, \star \star}$ \\ ${ }^{1}$ Institute of Computer Science, Faculty of Science, P. J. Šafárik University in Košice, Slovakia
}

\begin{abstract}
Recently an unexpected approximation property between polynomials of degree three and four was revealed within the framework of two-part approximation models in 2-norm, Chebyshev norm and Holladay seminorm. Namely, it was proved that if a twocomponent cubic Hermite spline's first derivative at the shared knot is computed from the first derivative of a quartic polynomial, then the spline is a clamped spline of class $C^{2}$ and also the best approximant to the polynomial.

Although it was known that a $2 \times 2$ component uniform bicubic Hermite spline is a clamped spline of class $C^{2}$ if the derivatives at the shared knots are given by the first derivatives of a biquartic polynomial, the optimality of such approximation remained an open question.

The goal of this paper is to resolve this problem. Unlike the spline curves, in the case of spline surfaces it is insufficient to suppose that the grid should be uniform and the spline derivatives computed from a biquartic polynomial. We show that the biquartic polynomial coefficients have to satisfy some additional constraints to achieve optimal approximation by bicubic splines.
\end{abstract}

\section{Introduction}

Lately a surprising property of polynomials within the framework of two-part models in case of function approximation has been found. Papers [1], [2] show that a two-component uniform cubic Hermite spline curve of class $C^{1}$ by its definition will be also of class $C^{2}$, if its coefficients are computed from a corresponding quartic polynomial. This relation was later confirmed for $2 \times 2$ component bicubic splines and biquartic polynomials [3]. However, unlike the two-component spline curve, the resulting four component spline surface is not automatically the best approximant to the biquartic polynomial.

The goal of this paper is to show that the coefficients of the biquartic polynomial have to satisfy some constraints to achieve optimal approximation by bicubic splines.

Let define the inputs, the given and computed coefficients to construct a $2 \times 2$ spline surface.

Consider a grid $\left[u_{0}, u_{1}, u_{2}\right] \times\left[v_{0}, v_{1}, v_{2}\right]$. According to [4], [5], a spline surface $S \equiv S(x, y)$ is defined by the given values

$$
z_{i, j}, \quad i=0,1,2, \quad j=0,1,2
$$

\footnotetext{
^e-mail: viliam.kacala@student.upjs.sk

$\star \star$ e-mail: csaba.torok@upjs.sk
} 
at the equispaced grid-points and the given first order directional derivatives

$$
d_{i, j}^{x}, \quad i=0,2, \quad j=0,1,2
$$

at the boundary verticals,

$$
d_{i, j}^{y}, \quad i=0,1,2, \quad j=0,2
$$

at the boundary horizontals and the cross derivatives

$$
d_{i, j}^{x, y}, \quad i=0,2, \quad j=0,2
$$

at the four corners of the grid.

To compute a bicubic clamped spline surface $S$ of class $C^{2}$ one needs to define each component of a quadruple $\left[z_{i, j}, d_{i, j}^{x}, d_{i, j}^{y}, d_{i, j}^{x, y}\right]$ at every grid-point $\left[u_{i}, v_{j}\right]$, with the properties

$$
z_{i, j}=S\left(u_{i}, v_{j}\right), \quad d_{i, j}^{x}=\frac{\partial S\left(u_{i}, v_{j}\right)}{\partial x}, \quad d_{i, j}^{y}=\frac{\partial S\left(u_{i}, v_{j}\right)}{\partial y}, \quad d_{i, j}^{x, y}=\frac{\partial^{2} S\left(u_{i}, v_{j}\right)}{\partial x \partial y},
$$

such that the adjacent spline segments would be twice continuously differentiable. This can be achieved using either de Boor's algorithm [4], [6] or a newer, computationally more efficient approach based on an interrelation between bicubic splines and biquartic polynomials [3].

For the sake of completeness we will define the relationship between splines and biquartic polynomials.

\section{Definition 1 The Hermite spline}

$$
S(x, y)= \begin{cases}S_{0,0}(x, y) & \text { for }(x, y) \in\left[u_{0}, u_{1}\right] \times\left[v_{0}, v_{1}\right], \\ S_{0,1}(x, y) & \text { for }(x, y) \in\left[u_{0}, u_{1}\right] \times\left[v_{1}, v_{2}\right], \\ S_{1,0}(x, y) & \text { for }(x, y) \in\left[u_{1}, u_{2}\right] \times\left[v_{0}, v_{1}\right], \\ S_{1,1}(x, y) & \text { for }(x, y) \in\left[u_{1}, u_{2}\right] \times\left[v_{1}, v_{2}\right]\end{cases}
$$

is based on the biquartic polynomial

$$
P(x, y)=\sum_{i=0}^{4} \sum_{j=0}^{4} a_{i, j} x^{i} y^{j}, \quad a_{4,4} \neq 0,
$$

if the coefficients of $S$ are computed from $P$ according to

$$
\begin{gathered}
S\left(u_{i}, v_{j}\right)=z_{i, j}=P\left(u_{i}, v_{j}\right), \quad i, j=0,1,2, \quad \frac{\partial S\left(u_{i}, v_{j}\right)}{\partial x}=d_{i, j}^{x}=\frac{\partial P\left(u_{i}, v_{j}\right)}{\partial x}, \quad i=0,2, j=0,1,2, \\
\frac{\partial S\left(u_{i}, v_{j}\right)}{\partial y}=d_{i, j}^{y}=\frac{\partial P\left(u_{i}, v_{j}\right)}{\partial y}, \quad i=0,1,2, j=0,2, \quad \frac{\partial^{2} S\left(u_{i}, v_{j}\right)}{\partial x \partial y}=d_{i, j}^{x, y}=\frac{\partial^{2} P\left(u_{i}, v_{j}\right)}{\partial x \partial y}, \quad i, j=0,2 .
\end{gathered}
$$

It is proved in [3] that $S$ will be of class $C^{2}$ if it is based on any biquartic polynomial and the grid $\left[u_{0}, u_{1}, u_{2}\right] \times\left[v_{0}, v_{1}, v_{2}\right]$ is uniform. The task is to find additional constraints under which the $2 \times 2$-component spline $S=\left\{S_{0,0}, S_{1,0}, S_{0,1}, S_{1,1}\right\}$ will be an optimal approximant to the biquartic polynomial as well. 


\section{Approximation optimality}

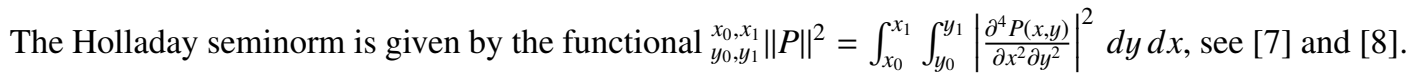

Theorem 1 Consider a grid $\left[u_{0}, u_{1}, u_{2}\right] \times\left[v_{0}, v_{1}, v_{2}\right]$ with $u_{0}<u_{1}<u_{2}, v_{0}<v_{1}<v_{2}$ and a biquartic polynomial $P$. Then the $2 \times 2$-component Hermite spline $S=\left\{S_{0,0}, S_{1,0}, S_{0,1}, S_{1,1}\right\}$ will be of class $C^{2}$ and the best approximant to $P$ in the Holladay seminorm, if $S$ is based on $P$ and

$$
u_{1}=\frac{1}{2}\left(u_{0}+u_{2}\right), \quad v_{1}=\frac{1}{2}\left(v_{0}+v_{2}\right), \quad 0=a_{2,4}=a_{4,2}=a_{3,4}=a_{4,3},
$$

\section{Proof:}

We have only to prove that ${ }_{v_{0}, v_{2}}^{u_{0} u_{2}}\|P-S\|^{2}$ attains its minimum under the assumptions of the theorem. First we derive the stationary point and then we find the conditions under which the norm attains its minimum in the stationary point.

The approximation error of the four-component Hermite spline in Holladay seminorm is (see [3])

$$
\begin{aligned}
N & ={ }_{v_{0}, u_{2}}^{u_{0}, v_{2}}\|P-S\|^{2}={ }_{v_{0}, u_{1}}^{u_{0}, u_{1}}\left\|P-S_{0,0}\right\|^{2}+{ }_{v_{1}, v_{2}}^{u_{0}, u_{1}}\left\|P-S_{0,1}\right\|^{2}++_{v_{0}, v_{1}}^{u_{1}, u_{2}}\left\|P-S_{1,0}\right\|^{2}++_{v_{1}, v_{2}}^{u_{1}, u_{2}}\left\|P-S_{1,1}\right\|^{2} \\
& ={ }_{v_{0}, u_{1}}^{u_{0}}\left\|\mathrm{ZA}\left(x, y, u_{0}, u_{1}, v_{0}, v_{1}\right)\right\|^{2}+{ }_{v_{1}, v_{2}}^{u_{0}, u_{2}}\left\|\mathrm{Z} A\left(x, y, u_{0}, u_{1}, v_{1}, v_{2}\right)\right\|^{2} \\
& +{ }_{v_{1}, u_{2}}^{u_{v_{0}}, u_{1}}\left\|\mathrm{ZA}\left(x, y, u_{1}, u_{2}, v_{0}, v_{1}\right)\right\|^{2}+{ }_{v_{1}, v_{2}}^{u_{1}, u_{2}}\left\|\mathrm{ZA}\left(x, y, u_{1}, u_{2}, v_{1}, v_{2}\right)\right\|^{2},
\end{aligned}
$$

where, e.g.,

$$
\begin{aligned}
Z A\left(x, y, x_{0}, x_{1}, y_{0}, y_{1}\right) & =Z\left(x, x_{0}, x_{1}\right)\left(a_{4,0}+a_{4,1} y+a_{4,2} y^{2}+a_{4,3} y^{3}\right)+ \\
& Z\left(y, y_{0}, y_{1}\right)\left(a_{0,4}+a_{1,4} x+a_{2,4} x^{2}+a_{3,4} x^{3}\right)+ \\
& a_{4,4}\left[Z\left(x, x_{0}, x_{1}\right) \sigma\left(y, y_{0}, y_{1}\right)+Z\left(y, y_{0}, y_{1}\right) \sigma\left(x, x_{0}, x_{1}\right)+Z\left(x, x_{0}, x_{1}\right) Z\left(y, y_{0}, y_{1}\right)\right],
\end{aligned}
$$

and $Z\left(t, t_{0}, t_{1}\right)=\left(t-t_{0}\right)^{2}\left(t-t_{1}\right)^{2}, \quad \sigma\left(t, t_{0}, t_{1}\right)=\left(t\left(t_{0}+t_{1}\right)-t_{0} t_{1}\right) \cdot\left(2 t^{2}-t\left(t_{0}+t_{1}\right)+t_{0} t_{1}\right)$.

Now we compute the partial derivatives of $N$

$$
\begin{aligned}
N_{u_{1}} & =\frac{\partial N\left(x, y, u_{0}, u_{1}, u_{2}, v_{0}, v_{1}, v_{2}\right)}{\partial u_{1}}=-16\left(u_{0}-u_{2}\right)\left(u_{2}+u_{0}-2 u_{1}\right) \cdot\left(u_{0}^{2}-2 u_{0} u_{1}+2 u_{1}^{2}\right)\left(v_{0}-v_{2}\right) R_{x}, \\
N_{v_{1}} & =\frac{\partial N\left(x, y, u_{0}, u_{1}, u_{2}, v_{0}, v_{1}, v_{2}\right)}{\partial v_{1}}=-16\left(v_{0}-v_{2}\right)\left(v_{2}+v_{0}-2 v_{1}\right) \cdot\left(v_{0}^{2}-2 v_{0} v_{1}+2 v_{1}^{2}\right)\left(u_{0}-u_{2}\right) R_{y}, \\
N_{u_{1}, v_{1}} & =\frac{\partial^{2} N\left(x, y, u_{0}, u_{1}, u_{2}, v_{0}, v_{1}, v_{2}\right)}{\partial u_{1} \partial v_{1}} \\
& =-16 a_{5,5}^{2}\left(v_{0}-v_{2}\right)\left(v_{2}+v_{0}-2 v_{1}\right)\left(v_{0}^{2}-2 v_{0} v_{1}+2 v_{1}^{2}\right)\left(u_{0}-u_{2}\right)\left(u_{2}+u_{0}-2 u_{1}\right)\left(u_{0}^{2}-2 u_{0} u_{1}+2 u_{1}^{2}\right),
\end{aligned}
$$

where the expressions $R_{x}$ and $R_{y}$ depend on $v_{0}, v_{1}, v_{2}, a_{4,2}, a_{4,3}, a_{4,4}$ and $u_{0}, u_{1}, u_{2}, a_{2,4}, a_{3,4}, a_{4,4}$, respectively. From the first derivatives $N_{u_{1}}$ and $N_{v_{1}}$ we get that a stationary point located at

$$
\left[u_{1}, v_{1}\right]=\left[\frac{1}{2}\left(u_{2}+u_{0}\right), \frac{1}{2}\left(v_{2}+v_{0}\right)\right] \text {. }
$$


Since $N_{u_{1}}$ and $N_{v_{1}}$ have no other real roots, $\left[u_{1}, v_{1}\right]$ denotes the location of a global extremum. To decide whether this is a point of minimum or maximum, the Hessian $H=N_{u_{1}, u_{1}} N_{v_{1}, v_{1}}-\left(N_{u_{1}, v_{1}}\right)^{2}$ at point $\left[u_{1}, v_{1}\right]$ is to be computed. Since $N_{u_{1}, v_{1}}=0$ at the stationary point, see above, after the evaluation of $N_{u_{1}, u_{1}}$ and $N_{v_{1}, v_{1}}$, the substitution of $u_{1}, v_{1}$ and some algebraic transformations, the following expression is obtained for $H$,

$$
H=\psi\left(u_{0}, u_{2}, a_{4,2}, a_{4,3}, a_{4,4}\right) \psi\left(v_{0}, v_{2}, a_{2,4}, a_{3,4}, a_{4,4}\right),
$$

where

$$
\begin{aligned}
\psi\left(t_{0}, t_{2}, b_{0}, b_{1}, b_{2}\right) & =16 b_{0}^{2}+\left(48\left(t_{0}+t_{2}\right) b_{1}+64\left(t_{0}^{3}+t_{0} t_{2}+t_{2}^{2}\right) b_{2}\right) b_{0}+48\left(t_{0}^{2}+t_{0} t_{2}+t_{2}^{2}\right) b_{1}^{2} \\
& +144\left(t_{0}^{3}+t_{0}^{2} t_{2}+t_{0} t_{2}^{2}+t_{2}^{3}\right) b_{2} b_{1}+\left(115 t_{0}^{4}+116 t_{0}^{3} t_{2}^{1}+114 t_{0}^{2} t_{2}^{2}+116 t_{0}^{1} t_{2}^{3}+115 t_{2}^{4}\right) b_{2}^{2} .
\end{aligned}
$$

Let $0=a_{2,4}=a_{4,2}=a_{3,4}=a_{4,3}$. Then

$$
H=\psi\left(u_{0}, u_{2}, 0,0, a_{4,4}\right) \psi\left(v_{0}, v_{2}, 0,0, a_{4,4}\right)
$$

where

$$
\psi\left(t_{0}, t_{2}, 0,0, b_{2}\right)=\left(115 t_{0}^{4}+116 t_{0}^{3} t_{2}^{1}+114 t_{0}^{2} t_{2}^{2}+116 t_{0}^{1} t_{2}^{3}+115 t_{2}^{4}\right) b_{2}^{2} .
$$

Since the quartic polynomial $\psi\left(t_{a}, t_{c}, 0,0, a_{4,4}\right)$ has no real root and is positive at least at one point, it results that $\psi\left(t_{a}, t_{c}, 0,0, a_{4,4}\right)>0$. Hence it follows immediatelly that the Hessian $H>0$, which means that under the assumption (1) the approximation error in Holladay seminorm $N$ has global minimum at the point $\left[u_{1}, v_{1}\right]=\left[\frac{1}{2}\left(u_{2}+u_{0}\right), \frac{1}{2}\left(v_{2}+v_{0}\right)\right]$.

Remark 1 The conditions on $a_{i, j}$ in (1) can be expressed using function values and derivatives as well, however, not uniquely. It follows from the theorem that, in general a bicubic spline, cannot optimally approximate even the nearest higher degree quartic polynomial.

\section{Summary}

We derived the constraints under wich a $2 \times 2$-component interpolating bicubic spline of class $C^{2}$ optimally approximates a biquartic polynomial in Holladay seminorm.

\section{Acknowledgement}

This work was partially supported by projects Technicom ITMS 26220220182 and APVV-15-0091 Effective algorithms, automata and data structures.

\section{References}

[1] Cs. Török, Matematicheskoe modelirovanie 26, 11, 33-36 (2014)

[2] J. Buša, L. Miňo, and Cs. Török, in Proceedings of 1st year of the Spring School of Doctoral Students (UPJŠ, Košice, Slovakia, 2015)

[3] L. Miňo, I. Szabó, and Cs. Török, Open Computer Science 6, 1, 1-7 (2016)

[4] C. de Boor, Journal of Mathematics and Physics 41 (3), 212-218 (1962)

[5] E.V. Shikin and A.I. Plis, Handbook on Splines for the User (CRC Press, 1995) 141 pp.

[6] D. Salomon, Curves and Surfaces for Computer Graphics (Springer, 2006), 1-460

[7] Advanced Course on FAIRSHAPE (Editors J. Hoscheck and P. Kaklis, B. G. Teubner Stuttgart, 1996) $210 \mathrm{pp}$.

[8] J. Stoer and R. Bulirsch, Introduction to numerical analysis (Springer, 2002) 99 pp. 